\title{
Putting Population Health into Action: A Series of Integrated Perspectives
}

Anu Melville, Lynn Eckendorf, and Todd Ellis

Editor's note: This is the first in a series of articles, where Todd Ellis and colleagues will show how various population health "workstreams" are integrated real-time, and how they can help manage outcomes and costs.

This series of articles will present a comprehensive view of one patient's journey within a healthcare system following a cerebral vascular accident (stroke). The authors will delve into specific activities of each workstream, how they work together, and how the patient and family interact with caregivers during this journey.

Using the case history format, our goal is to provide an opportunity for healthcare professionals to reflect on their organization and its use of telemedicine, with emphasis on how it currently manages population health and outcomes through the continuum of care. We will show how payment for outcomes (value-based care) is enabled by integrating population health management workstreams, in many cases leveraging components you already have.

\section{An Elderly African-American Male}

The patient is a 48-year-old African-American male with a past medical history that is significant a 15-year history of type 2 diabetes mellitus and stroke in the past year. The latest hemoglobin A1c (glycated hemoglobin) blood level is elevated (7.0\%; normal: $<5.7 \%$ ), with no change in the past 3 months. There is evidence of diabetic retinopathy. He has hypertension $(200 / 120 \mathrm{mmHg})$ with a creatinine of $3.2 \mathrm{mg} / \mathrm{dL}$.

His primary care physician monitors his diabetes, and reinforces the importance of prescribed diet and exercise recommendations. He is to follow-up with foot and eye exams and return to the clinic in 3 months. An English-speaking family member usually accompanies him or is on the phone with him during physician visits. He has added risk factors for stroke (ie, high blood pressure, previous stroke, diabetes, elderly, African American) and is encouraged to recommit to a healthy lifestyle, with monitoring. 


\section{Stoke}

Despite monitoring and lifestyle changes, one morning he experiences sudden numbness along the right side of his body, blurred vision, lose of balance, and falling as he tried to get out of bed.

Fortunately, his daughter arrives 30 minutes later to check up on him. Finding him on the floor, she phones the physician's office and is instructed to call 911. EMS arrive with transport to the nearest emergency department (ED) for evaluation. Unable to communicate clearly, the ED staff retrieves his record from the hospital's electronic medical record (EMR) system. After a teleconference with a neurologist and interpreter, the stroke protocol is initiated (ie, notification of the stroke team, CT or MRI, chest Xray, labs and tissue plasminogen activator (tPA) administration, admission to the intensive care).

After a time, he is transferred to the medical/surgical unit for additional care and monitoring. When ready, he is discharged to a rehabilitation facility, then home for home-based care and follow-up.

\section{Next Time}

The second installment in this series will show how Telehealth and Technology are used to enable patient care activities, and provide data that are subsequently analyzed and used to provide care.

In the third installment, Data Integration and Analytics will support developing care plans and analyzing outcomes.

The fourth installment will look over the shoulder of a Care Manager, showing how all the elements are connected to proactively care for our patient across the care continuum and produce desired outcomes.

In the fifth installment, we will discuss the impact of Financial Planning and Operations as the context for identifying care and cost efficiencies. 
Finally, the view from the Patient/Family perspective will wrap up how all the population health components are distilled into what the patient/family experiences throughout the journey.

We invite you to join us.

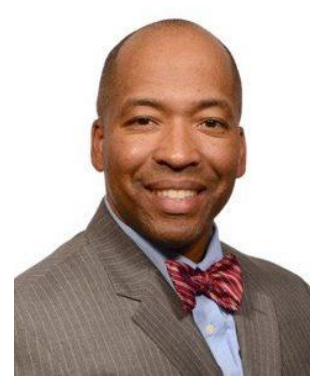

Todd Ellis

Series Authors: Anu Melville is Manager Advisory Healthcare and Life Sciences, KPMG; Lynn Eckendorf is Manager Advisory Healthcare and Life Sciences, KPMG, and Todd Ellis is Principal, KPMG.

Tags: African-American mail, care manager, financial planning and operations, homebased car, population health workstreams, rehabilitation facility, Telehealth, type 2 diabetes 